\title{
Averaged Template Matching Equations
}

\author{
Anil N. Hirani, Jerrold E. Marsden, and James Arvo \\ California Institute of Technology, Pasadena, CA, USA.
}

\begin{abstract}
By exploiting an analogy with averaging procedures in fluid dynamics, we present a set of averaged template matching equations. These equations are analogs of the exact template matching equations that retain all the geometric properties associated with the diffeomorphism group, and which are expected to average out small scale features and so should, as in hydrodynamics, be more computationally efficient for resolving the larger scale features. From a geometric point of view, the new equations may be viewed as coming from a change in norm that is used to measure the distance between images. The results in this paper represent first steps in a longer term program: what is here is only for binary images and an algorithm for numerical computation is not yet operational. Some suggestions for further steps to develop the results given in this paper are suggested.
\end{abstract}

\section{Introduction}

\subsection{Previous Work}

Deformable template matching is a technique for comparing images with applications in computer vision, medical imaging and other fields. It has been reported on extensively in the literature. See for example, Younes (2000), Trouvé (1995, 1998), Grenander and Miller (1998) and the references therein.

Template matching is based on the notion of computing a deformation induced distance between two images. The "energy" required to do a deformation that takes one image to the other defines the distance between them. The deformations are often taken to be diffeomorphisms of the image rectangle, i.e smooth maps with smooth inverse. The energy can be defined using various metrics on the space of diffeomorphisms. In addition to diffeomorphisms, which are merely a change of coordinates of the underlying image rectangle, one can also allow changes to the pixel values. Trouvé $(1995,1998)$ develops such a theory and gives several numerical examples. He gives conditions on the metric that are sufficient to make the space of deformations a complete metric space. He works with a subgroup of homeomorphisms as the space of deformations and allows pixel value changes by using a semidirect product with a group that acts on the pixel values. The paper by Dupuis, Grenander and Miller (1998) also derives conditions for existence of template matching solutions.

Recently a partial differential equation for template matching was derived by Ratnanather, Baigent, Mumford and Miller (2000), for both exact and inexact matching. In exact matching the two images being compared have to be 
diffeomorphic and in inexact matching they need not be. Their derivation was done using Euler-Poincaré reduction theory and also using classical calculus of variations. For an early version, which does not use the Euler-Poincaré theory, see Mumford (1998b). For Euler-Poincaré reduction theory, see Marsden and Ratiu (1999, Chapters 1 and 13). This technique is useful for computing EulerLagrange equations when the Lagrangian is invariant under the action of some Lie group. For example, it is possible to do a variational derivation of the Euler equations of rigid bodies and fluid mechanics using Euler-Poincaré reduction theory.

In their most general form as given in Mumford (1998b), the exact template matching equations (TME) depend on the choice of a self-adjoint operator that appears in the definition of the metric on the group of diffeomorphisms. When this metric is $\mathrm{L}^{2}$ we will refer to the equations as $\mathrm{L}^{2}$-TME.

\subsection{Contributions}

In this paper we derive the isotropic averaged template matching equations, $\left(\mathrm{H}_{\alpha}^{1}\right.$-ATME), which we hope will be a version of the exact template matching equations that average out small scale features, yet retain the larger scale features. The $\mathrm{H}_{\alpha}^{1}$ refers to a weighted Sobolev metric that we use instead of the $\mathrm{L}^{2}$ metric on the group of diffeomorphisms.

Thus the $\mathrm{H}_{\alpha}^{1}$-ATME are derived by making a special choice for the self-adjoint operator that appears in the derivation of Ratnanather, Baigent, Mumford and Miller (2000). We expect that the averaged equations and even their anisotropic counterparts may also be of interest in computer vision. These might allow template matching while ignoring features smaller than a chosen size ( $\alpha$ in equation (13)). The $\mathrm{H}_{\alpha}^{1}$-ATME derivation was inspired by recent work on Lagrangian averaged equations in fluid mechanics as described in Marsden and Shkoller (2001) and references there in.

By analogy with fluid mechanics the $\mathrm{H}_{\alpha}^{1}$-ATME may be much more amenable to numerical solution than the $\mathrm{L}^{2}$-TME. Finally, by allowing the ignorable feature size to vary it may be possible to perform template matching more robustly using a multiscale approach.

\subsection{Overview}

We first set up the framework of template matching. For this paper, the main task of template matching reduces to defining distance between binary images. After describing the framework we give the definitions and facts that are needed for our derivation. These preliminaries include a brief summary of Euler-Poincaré reduction in Section 3.3. Before giving a derivation of $\mathrm{H}_{\alpha}^{1}$-ATME we repeat the derivation of the TME of Mumford (1998b) for the special case of the $\mathrm{L}^{2}$ metric. The main result in this paper is the derivation of the isotropic averaged template matching equations for the exact matching case and this derivation is in Section 6. 


\section{Our Framework}

The basic element of template matching is the computation of a distance between two images and the computation of a deformation that takes one image to the other. For concreteness and simplicity we will limit our attention to binary images, i.e. an image will be a characteristic function on some bounded open subset $M$ of $\mathbb{R}^{n}, n \geq 1$. Thus our space of images is $\mathcal{P}=\{f \mid f: M \rightarrow\{0,1\}\}$. We do the derivations for a general $n$ since those are just as easy as derivations for the case $n=2$. For $n=2, M$ will typically be a rectangle in the plane.

In order to define a metric on $\mathcal{P}$, given two images $f$ and $g$ one finds the "smallest" map $\varphi: M \rightarrow M$ such that $f=g \circ \varphi$. In Section 3.2 we show that this smallest map $\varphi$ induces a pseudometric on $\mathcal{P}$. This approach does not allow one to modify the range of the images, $\varphi$ is just a change of coordinates for $M$. A more general framework that allows one to modify the range of the images (i.e. modify the pixel values) by using semidirect products is described in Trouvé (1995).

In order to define the "smallest" map $\varphi$ mentioned above, one must define a metric on the space to which $\varphi$ belongs. In addition one has a choice of what space to use as the source of the maps $\varphi$. These choices and an analysis of their implications require extensive and subtle analysis that uses many mathematical tools. A nice discussion and use of these subtleties is in Trouve (1995). In this paper, to keep things simple, we will ignore these subtleties. We will take the space to which the maps $\varphi$ belong to be the space of all diffeomorphisms of $M$ fixing its boundary pointwise and denote this space by $\operatorname{Diff}(M)$.

\section{Preliminaries}

\subsection{Facts About $\operatorname{Diff}(M)$}

We will require some basic facts about $\operatorname{Diff}(M)$ and its tangent spaces which we state here, some without proof. We will ignore the difficulties associated with defining a differentiable structure on $\operatorname{Diff}(M)$. For details on this, see Ebin and Marsden (1970). See also Marsden and Ratiu (1999) for an elementary discussion. The most important fact is that a vector in the tangent space of $\operatorname{Diff}(M)$ is a vector field on $M$. This is stated more formally in Facts 1 and 2 below.

Fact 1. The tangent space of $\operatorname{Diff}(M)$ at the point $\varphi \in \operatorname{Diff}(M)$, is the space of all material (i.e Lagrangian) velocity vector fields $\boldsymbol{V}_{\varphi}$ over $\varphi$ on $M$ that vanish on the boundary $\partial M$ of $M$. This tangent space is denoted by $T_{\varphi}(\operatorname{Diff}(M))$.

Fact 2. The tangent space of $\operatorname{Diff}(M)$ at identity $e \in \operatorname{Diff}(M)$, i.e. $T_{e}(\operatorname{Diff}(M))$ is the space $\mathfrak{X}(M)$ of all spatial (i.e Eulerian) velocity vector fields on $M$ that vanish on the boundary $\partial M$ of $M$.

We also need some facts about the tangent map (derivative) of the action of $\operatorname{Diff}(M)$ acting on itself. Let $\operatorname{Diff}(M)$ act on itself on the right by function 
composition. Thus for $\varphi, \eta \in \operatorname{Diff}(M)$ the right action of $\eta$ on $\varphi$ is $\varphi \cdot \eta=$ $R_{\eta}(\varphi)=\varphi \circ \eta$, where $R_{\eta}$ denotes right multiplication of the argument by $\eta$. Now we compute the tangent lifted action, i.e $T R_{\eta}$ which is the derivative of the right action described above. We show that

Fact 3. If $R_{\eta}$ is the right action defined above, then its derivative is

$$
T_{\varphi} R_{\eta}\left(\boldsymbol{V}_{\varphi}\right)=\boldsymbol{V}_{\varphi} \circ \eta
$$

for all $\boldsymbol{V}_{\varphi} \in T_{\varphi}(\operatorname{Diff}(M))$. This is called the right action by $\eta$ on $\boldsymbol{V}_{\varphi}$.

We recall the proof of this standard fact for the reader's convenience.

Proof. The proof essentially consists of checking definitions. Let $\varphi_{t} \subset \operatorname{Diff}(M)$ be a smooth curve such that $\varphi_{0}=\varphi$ and $d /\left.d t\right|_{t=0} \varphi_{t}=\boldsymbol{V}_{\varphi}$. Thus for every $X \in$ $M, \partial /\left.\partial t\right|_{t=0} \varphi(X, t)=\boldsymbol{V}_{\varphi}(X)$. Then by definition of derivative $T_{\varphi} R_{\eta}\left(\boldsymbol{V}_{\varphi}\right)=$ $d /\left.d t\right|_{t=0}\left(\varphi_{t} \circ \eta\right)$. Thus, $T_{\varphi} R_{\eta}\left(\boldsymbol{V}_{\varphi}\right)(X)=d /\left.d t\right|_{t=0} \varphi_{t}(\eta(X))=\boldsymbol{V}_{\varphi}(\eta(X))$.

Definition 1. An inner product on $\operatorname{Diff}(M)$ is said to be right invariant under action by $\operatorname{Diff}(M)$ if $\left(\boldsymbol{V}_{\varphi}, \boldsymbol{U}_{\varphi}\right)=\left(T_{\varphi} R_{\eta}\left(\boldsymbol{V}_{\varphi}\right), T_{\varphi} R_{\eta}\left(\boldsymbol{U}_{\varphi}\right)\right)=\left(\boldsymbol{V}_{\varphi} \circ \eta, \boldsymbol{U}_{\varphi} \circ \eta\right)$ for all $\varphi, \eta \in \operatorname{Diff}(M)$ and $\boldsymbol{V}_{\varphi}, \boldsymbol{U}_{\varphi} \in T_{\varphi}(\operatorname{Diff}(M))$. Here $(\cdot, \cdot)$ denotes an inner product on $\operatorname{Diff}(M)$.

\subsection{Pseudometric Using Diffeomorphisms}

We induce a pseudometric (Abraham, Marsden and Ratiu (1988)) on the space of images $\mathcal{P}$ from a metric defined on $\operatorname{Diff}(M)$ as follows.

Definition 2. The positive valued function $\mathrm{d}_{\mathcal{P}}: \mathcal{P} \times \mathcal{P} \rightarrow \mathbb{R}$, is called a pseudometric induced on $\mathcal{P}$ from $\operatorname{Diff}(M)$, if for any $f, g \in \mathcal{P}$

$$
\mathrm{d}_{\mathcal{P}}(f, g)=\inf \{d(e, \varphi) \mid \varphi \in \operatorname{Diff}(M) \text { and } f=g \circ \varphi\},
$$

where $e$ is the identity diffeomorphism, $d(e, \varphi)$ is the geodesic distance between $e$ and $\varphi$ and inf stands for infimum, or greatest lower bound.

As usual, the geodesic distance on $\operatorname{Diff}(M)$, is defined in terms of the inner product on the tangent spaces of $\operatorname{Diff}(M)$, i.e. the Riemannian metric on $\operatorname{Diff}(M)$. One must prove that $d_{\mathcal{P}}$ as defined above is actually a pseudometric, i.e. that it satisfies the symmetry and triangle inequality properties as well as the property that $\mathrm{d}_{\mathcal{P}}(f, f)=0$ for all $f \in \mathcal{P}$. This is stated in the following Fact 4. See Miller and Younes (1999) for a sketch of a proof or Hirani, Marsden and Arvo (2001), which is the technical report version of the present paper, for a more detailed proof.

Fact 4. If the Riemannian metric on $\operatorname{Diff}(M)$ is right invariant under action by $\operatorname{Diff}(M)$, then the function $\mathrm{d}_{\mathcal{P}}$ of Definition 2 satisfies the pseudometric axioms, namely that

1. $\mathrm{d}_{\mathcal{P}}(f, f)=0$ for all $f \in \mathcal{P}$; 
2. $\mathrm{d}_{\mathcal{P}}(f, g)=\mathrm{d}_{\mathcal{P}}(g, f)$ for all $f, g \in \mathcal{P}$ (symmetry) ; and

3. $\mathrm{d}_{\mathcal{P}}(f, h) \leq \mathrm{d}_{\mathcal{P}}(f, g)+\mathrm{d}_{\mathcal{P}}(g, h)$ for all $f, g, h \in \mathcal{P}$ (triangle inequality).

Furthermore, if the infimum in Definition 2 is achieved, then $\mathrm{d}_{\mathcal{P}}$ is a metric on $\mathcal{P}$, in which case property 1 becomes

$$
\text { 1. } \mathrm{d}_{\mathcal{P}}(f, g)=0 \Longleftrightarrow f=g \text { (definiteness) . }
$$

One can also show the same result when right invariance is replaced by left invariance or bi-invariance.

Thus to compute $\mathrm{d}_{\mathcal{P}}(f, g)$ we need to find the smallest diffeomorphism $\varphi$ in $\operatorname{Diff}(M)$ such that $f=g \circ \varphi$. The definition of smallest depends on the chosen metric on $\operatorname{Diff}(M)$. The typical strategy for this is to find a geodesic on $\operatorname{Diff}(M)$, from the identity map to the unknown diffeomorphism $\varphi$. The unknown diffeomorphism satisfies the constraint $f=g \circ \varphi$. Any $\operatorname{such} \varphi$ will be the smallest, since it will be the diffeomorphism closest to identity, which also satisfies $f=$ $g \circ \varphi$.

There may or may not be many such smallest diffeomorphisms, but it is sufficient to find one, in order to solve the template matching problem. The lack of uniqueness, if present, may have practical implications for numerical solvers, which is an issue we have not yet addressed. Moreover, there may not exist any such $\varphi$; for example this is the case when the image corresponding to $f$ is not homemorphic to that corresponding to $g$. The existence issue also requires further investigation. Trouvé $(1995,1998)$ gives conditions that a metric must satisfy for existence and uniqueness of minimizers in inexact matching.

\subsection{Euler-Poincaré Reduction}

We now recall some facts about Euler-Poincaré reduction theorem that we will need. For details, see Chapter 13 of Marsden and Ratiu (1999). Euler-Poincaré reduction is useful in mechanics. For example, it is possible to do a variational derivation of the Euler equations of rigid bodies and fluid mechanics using EulerPoincaré reduction theory. Consider a Lagrangian $L$ i.e. a map $L: T(\operatorname{Diff}(M)) \rightarrow$ $\mathbb{R}$, so $L$ is a function of $\varphi \in \operatorname{Diff}(M)$ and $\dot{\varphi} \in T_{\varphi}(\operatorname{Diff}(M))$. If this Lagrangian is invariant under right action by $\operatorname{Diff}(M)$ then we can use the Euler-Poincaré reduction theorem (Marsden and Ratiu (1999) Theorem 13.5.3). According to this theorem, the following two statements are equivalent :

1. The variational principle $\delta \int_{a}^{b} L(\varphi(X, t), \dot{\varphi}(X, t)) d t=0$ holds for variations of curves $\varphi(X, t)$ with fixed end points, i.e. for $\delta \varphi(X, a)=\delta \varphi(X, b)=$ $0 \forall X \in M$;

2. The variational principle $\delta \int_{a}^{b} l(\boldsymbol{u}(x, t)) d t=0$ holds on $\mathfrak{X}(M)$, i.e. on the tangent space at the identity of $\operatorname{Diff}(M)$, using variations of the form $\delta \boldsymbol{u}=$ $\dot{\boldsymbol{w}}+[\boldsymbol{w}, \boldsymbol{u}]_{L}$. This is called the reduced variational principle.

Here $\dot{\varphi}(X, t)=\partial / \partial t \varphi(X, t)$ (keeping $X$ fixed). The vector $\boldsymbol{u}$ is the tangent vector $\dot{\varphi}$ moved to identity $e \in \operatorname{Diff}(M)$ by right action by $\varphi_{t}^{-1}$, i.e. $\boldsymbol{u}=\dot{\varphi} \circ$ 
$\varphi_{t}^{-1}$. Subscript $t$ here denotes fixed time $t$ not derivative w.r.t $t$. More precisely, if $x=\varphi(X, t)=\varphi_{t}(X)$ then $\boldsymbol{u}(x, t)=\dot{\varphi}\left(\varphi_{t}^{-1}(x), t\right)$. By this we mean that first the time derivative of $\varphi(X, t)$ is computed keeping $X$ fixed and then one substitutes $X=X(x, t)=\varphi_{t}^{-1}(x)$ into the resulting expression. The function $l: T_{e}(\operatorname{Diff}(M)) \rightarrow \mathbb{R}$ is simply the restriction of $L$ to the tangent space at identity $e$.

The vector $\boldsymbol{w}$ is the vector $\delta \varphi$ moved to identity in $\operatorname{Diff}(M)$. Thus $\boldsymbol{w}=\delta \varphi_{t} \circ$ $\varphi_{t}^{-1}$. The notation $[\boldsymbol{w}, \boldsymbol{u}]_{L} \equiv(\boldsymbol{u} \cdot \nabla) \boldsymbol{w}-(\boldsymbol{w} \cdot \nabla) \boldsymbol{u}$ is the Jacobi Lie bracket. Note that $\boldsymbol{w}(x, a)=\boldsymbol{w}(x, b)=0 \quad \forall x \in M$ since $\delta \varphi(X, a)=\delta \varphi(X, b)=0 \forall X \in M$.

\subsection{Gauss-Green Theorem and its Corollary}

We will need some basic facts from vector calculus, for the derivation of the $\mathrm{L}^{2}$-TME and $\mathrm{H}_{\alpha}^{1}$-ATME. We state these facts here.

Fact 5. (Gauss-Green Theorem) Let $M$ be an open bounded subset of $\mathbb{R}^{n}$ and suppose that the boundary $\partial M$ is $C^{1}$. Suppose $u \in C^{1}(\bar{M})$. Then

$$
\int_{M} \frac{\partial u}{\partial x_{i}} d x=\int_{\partial M} u \nu^{i} d S
$$

for all $i=1, \ldots, n$ and where $\hat{\nu}=\left(\nu^{1}, \ldots, \nu^{n}\right)$ is the outward pointing unit normal field on $\partial M$.

A simple corollary of the Gauss-Green theorem is the following fact which we will use several times.

Fact 6. Let $M$ be an open bounded subset of $\mathbb{R}^{n}$ and suppose that $\partial M$ is $C^{1}$. Let $\mathbf{u}, \mathbf{v}, \mathbf{w}$ be vector fields on $\bar{M}$. Then

$$
\int_{M} \operatorname{div} \boldsymbol{v}\langle\boldsymbol{u}, \boldsymbol{w}\rangle+\langle\boldsymbol{u},(\boldsymbol{v} \cdot \nabla) \boldsymbol{w}\rangle+\langle(\boldsymbol{v} \cdot \nabla) \boldsymbol{u}, \boldsymbol{w}\rangle d x=\int_{\partial M}\langle\boldsymbol{u}, \boldsymbol{w}\rangle\langle\boldsymbol{v}, \hat{\nu}\rangle d S
$$

where $\hat{\nu}=\left(\nu^{1}, \ldots, \nu^{n}\right)$ is the outward pointing unit normal field of $\partial M$.

Proof. We will use the Gauss-Green theorem (Fact 5 ) stated above. By this theorem we have that for all $i, j$ in $\{1, \ldots, n\}, \int_{M} \partial / \partial x_{j}\left(u^{i} w^{i} v^{j}\right) d x=\int_{\partial M} u^{i} w^{i} v^{j} \nu^{j} d S$. Thus

$$
\sum_{i, j=1}^{n} \int_{M} u^{i} w^{i} \frac{\partial v^{j}}{\partial x_{j}}+u^{i} v^{j} \frac{\partial w^{i}}{\partial x_{j}}+w^{i} v^{j} \frac{\partial u^{i}}{\partial x_{j}} d x=\sum_{i, j=1}^{n} \int_{\partial M} u^{i} w^{i} v^{j} \nu^{j} d S,
$$

which proves equation (1).

\section{Metrics on $\operatorname{Diff}(M)$}

We need to define two different Riemannian metrics on Diff $(M)$, i.e. inner products on its tangent spaces. One is for the $\mathrm{L}^{2}$-TME derivation and the other one is for the $\mathrm{H}_{\alpha}^{1}$-ATME derivation. 
Definition 3. Let $\boldsymbol{V}_{\varphi}, \boldsymbol{U}_{\varphi} \in T_{\varphi}(\operatorname{Diff}(M))$, i.e. $\boldsymbol{V}_{\varphi}, \boldsymbol{U}_{\varphi}$ are tangent vectors, tangent to $\operatorname{Diff}(M)$ at the point $\varphi \in \operatorname{Diff}(M)$. Let $J(\varphi)(X)$ be the determinant of the derivative of (i.e. the Jacobian determinant of) $\varphi$ evaluated at point $X \in M$. The $\mathrm{L}^{2}$ Riemannian metric on $\operatorname{Diff}(M)$ we will use for the $\mathrm{L}^{2}-T M E$ derivation is defined as

$$
\left(\boldsymbol{V}_{\varphi}, \boldsymbol{U}_{\varphi}\right)_{\mathrm{L}^{2}} \equiv \int_{M}\left\langle\boldsymbol{V}_{\varphi}(X), \boldsymbol{U}_{\varphi}(X)\right\rangle J(\varphi)(X) d X
$$

Here $\langle\cdot, \cdot\rangle$ is the standard inner product on $\mathbb{R}^{n}$.

The $\mathrm{H}_{\alpha}^{1}$ metric on $\operatorname{Diff}(M)$ is defined by first defining it on the tangent space at identity and then extending it to all of $\operatorname{Diff}(M)$ right invariantly.

Definition 4. Let $\boldsymbol{v}, \boldsymbol{u}$ be vectors in the tangent space at identity $e \in \operatorname{Diff}(M)$ i.e. $\boldsymbol{v}, \boldsymbol{u} \in T_{e}(\operatorname{Diff}(M))$. For any $\alpha>0, \alpha \in \mathbb{R}$, define

$$
(\boldsymbol{v}, \boldsymbol{u})_{\mathrm{H}_{\alpha}^{1}} \equiv \int_{M}\langle\boldsymbol{v}(x), \boldsymbol{u}(x)\rangle+\alpha^{2} \sum_{i=1}^{n}\left\langle\mathbf{D}_{i} \boldsymbol{v}(x), \mathbf{D}_{i} \boldsymbol{u}(x)\right\rangle d x
$$

where $\mathbf{D}_{i} \boldsymbol{v}=\partial / \partial x_{i}(\boldsymbol{v}(x))$. The inner products inside the integral are the standard inner products (dot products) on $\mathbb{R}^{n}$.

To compute the inner product at a point $\varphi \in \operatorname{Diff}(M)$ different from identity, define $\left(\boldsymbol{V}_{\varphi}, \boldsymbol{U}_{\varphi}\right)_{\mathrm{H}_{\alpha}^{1}}=\left(\boldsymbol{V}_{\varphi} \circ \varphi^{-1}, \boldsymbol{U}_{\varphi} \circ \varphi^{-1}\right)_{\mathrm{H}_{\alpha}^{1}}$. Note that $\boldsymbol{V}_{\varphi} \circ \varphi^{-1}, \boldsymbol{U}_{\varphi} \circ \varphi^{-1} \in$ $T_{e}(\operatorname{Diff}(M))$ because right action by $\varphi^{-1}$ moves the vectors at $\varphi$ to identity on $\operatorname{Diff}(M)$. Thus we can use (3) to compute the inner product. Note that we defined the $\mathrm{L}^{2}$ inner product at a general point of $\operatorname{Diff}(M)$ but defined the $\mathrm{H}_{\alpha}^{1}$ inner product at identity and showed how it can be computed at a general point. This is done for simplicity. The expression for the $\mathrm{L}^{2}$ inner product at a general point is simple but not so for the $\mathrm{H}_{\alpha}^{1}$ inner product. We will write the corresponding norms as follows. The $\mathrm{L}^{2}$ norm of $\boldsymbol{U}_{\varphi} \in T_{\varphi}(\operatorname{Diff}(M))$ is written as $\left\|\boldsymbol{U}_{\varphi}\right\|_{\mathrm{L}^{2}} \equiv\left(\boldsymbol{U}_{\varphi}, \boldsymbol{U}_{\varphi}\right)_{\mathrm{L}^{2}}^{1 / 2}$ and similarly for the $\mathrm{H}_{\alpha}^{1}$ norm.

The geodesic distance between $\varphi_{a}$ and $\varphi_{b}$ on $\operatorname{Diff}(M)$ is defined as

$$
d\left(\varphi_{a}, \varphi_{b}\right) \equiv \inf \left\{\int_{a}^{b}(\dot{\varphi}(t), \dot{\varphi}(t))^{1 / 2} d t \mid \varphi(a)=\varphi_{a}, \varphi(b)=\varphi_{b}\right\}
$$

and the infimum is taken over all smooth parametric curves $\varphi:[a, b] \rightarrow \operatorname{Diff}(M)$ from $\varphi_{a}$ to $\varphi_{b}$. Here $(\cdot, \cdot)$ is any Riemannian metric on $\operatorname{Diff}(M)$. Thus $d\left(\varphi_{a}, \varphi_{b}\right)=$ $\int_{a}^{b}(\dot{\varphi}(t), \dot{\varphi}(t))^{1 / 2} d t$, where $\varphi$ is the curve between the endpoints that makes $\int_{a}^{b}(\dot{\varphi}(t), \dot{\varphi}(t))^{1 / 2} d t$ stationary.

The same curve makes the functional $\int_{a}^{b}(\dot{\varphi}(t), \dot{\varphi}(t)) d t$ stationary. This fact is sometimes stated as "minimizing length is the same as minimizing kinetic energy". One way to prove this fact is by computing the Euler- Lagrange equations for both the integrals and noting that the equations are the same. 


\subsection{Right Invariance of $\mathrm{L}^{2}$ Metric}

We now prove the right invariance property of the $\mathrm{L}^{2}$ metric in Definition 3 . This property is crucial for application of the Euler-Poincaré reduction theorem.

Claim 1. The metric defined by (2) is right invariant under action of the group $\operatorname{Diff}(M)$ acting on $\operatorname{Diff}(M)$, i.e. for any $\eta \in \operatorname{Diff}(M)$

$$
\left(T_{\varphi} R_{\eta}\left(\boldsymbol{V}_{\varphi}\right), T_{\varphi} R_{\eta}\left(\boldsymbol{U}_{\varphi}\right)\right)_{\mathrm{L}^{2}}=\left(\boldsymbol{V}_{\varphi}, \boldsymbol{U}_{\varphi}\right)_{\mathrm{L}^{2}}
$$

Proof. By the computation of the tangent lifted group action in Fact 3, this is equivalent to showing that $\left(\boldsymbol{V}_{\varphi} \circ \eta, \boldsymbol{U}_{\varphi} \circ \eta\right)_{\mathrm{L}^{2}}=\left(\boldsymbol{V}_{\varphi}, \boldsymbol{U}_{\varphi}\right)_{\mathrm{L}^{2}}$. The left hand side above is equal to $\int_{M}\left\langle\boldsymbol{V}_{\varphi}(\eta(X)), \boldsymbol{U}_{\varphi}(\eta(X))\right\rangle J(\varphi \circ \eta)(X) d X$. Note that the argument $\varphi \circ \eta$ of $J$ is the base point of $T_{\varphi} R_{\eta}\left(\boldsymbol{V}_{\varphi}\right)$ and $T_{\varphi} R_{\eta}\left(\boldsymbol{U}_{\varphi}\right)$ as required by the definition of the metric. Using chain rule for $J(\varphi \circ \eta)(X)$ the above integral becomes $\int_{M}\left\langle\boldsymbol{V}_{\varphi}(\eta(X)), \boldsymbol{U}_{\varphi}(\eta(X))\right\rangle J(\varphi)(\eta(X)) J(\eta)(X) d X$. Now use the change of variable $Y=\eta(X)$. By the change of variables theorem then $d Y=$ $J(\eta)(X) d X$ and the above integral becomes $\int_{\eta(M)}\left\langle\boldsymbol{V}_{\varphi}(Y), \boldsymbol{U}_{\varphi}(Y)\right\rangle J(\varphi)(Y) d Y$. Since $\eta(M)=M$ the above is equal to $\left(\boldsymbol{V}_{\varphi}, \boldsymbol{U}_{\varphi}\right)_{\mathrm{L}^{2}}$ as desired.

We now give the intuition behind the form of the $\mathrm{L}^{2}$ inner product. Specifically we address the question of why the Jacobian determinant term appears in the $\mathrm{L}^{2}$ inner product definition (Definition 2). It appears so that the inner product can be made right invariant. Right invariance of the metric on $\operatorname{Diff}(M)$ implies that the induced function $\mathrm{d}_{\mathcal{P}}$ is a pseudometric (Fact 4). Thus right invariance (or left- or bi-invariance for that matter) is a convenient assumption. Moreover, the distance between two images should not change if they are both distorted by the same change of variables. This also makes the requirement of invariance attractive.

\subsection{Right Invariance of $\mathrm{H}_{\alpha}^{1}$ Metric}

Since the $\mathrm{H}_{\alpha}^{1}$ metric was defined at identity and extended in a right invariant fashion, the check for right invariance is easy. For completeness we give it below.

Claim 2. The $\mathrm{H}_{\alpha}^{1}$ metric defined by (3) is right invariant under action of the group $\operatorname{Diff}(M)$ acting on $\operatorname{Diff}(M)$, i.e. for any $\eta \in \operatorname{Diff}(M)$

$$
\left(T_{\varphi} R_{\eta}\left(\boldsymbol{V}_{\varphi}\right), T_{\varphi} R_{\eta}\left(\boldsymbol{U}_{\varphi}\right)\right)_{\mathrm{H}_{\alpha}^{1}}=\left(\boldsymbol{V}_{\varphi}, \boldsymbol{U}_{\varphi}\right)_{\mathrm{H}_{\alpha}^{1}} .
$$

Proof. By Fact 3, it is enough to show that $\left(\boldsymbol{V}_{\varphi} \circ \eta, \boldsymbol{U}_{\varphi} \circ \eta\right)_{\mathrm{H}_{\alpha}^{1}}=\left(\boldsymbol{V}_{\varphi}, \boldsymbol{U}_{\varphi}\right)_{\mathrm{H}_{\alpha}^{1}}$ for all $\eta \in \operatorname{Diff}(M)$. But by definition of $\mathrm{H}_{\alpha}^{1}$,

$$
\begin{aligned}
\left(\boldsymbol{V}_{\varphi} \circ \eta, \boldsymbol{U}_{\varphi} \circ \eta\right)_{\mathrm{H}_{\alpha}^{1}} & =\left(\boldsymbol{V}_{\varphi} \circ \eta \circ(\varphi \circ \eta)^{-1}, \boldsymbol{U}_{\varphi} \circ \eta \circ(\varphi \circ \eta)^{-1}\right)_{\mathrm{H}_{\alpha}^{1}} \\
& =\left(\boldsymbol{V}_{\varphi}, \boldsymbol{U}_{\varphi}\right)_{\mathrm{H}_{\alpha}^{1}} .
\end{aligned}
$$




\section{Template Matching Equations}

Both the exact and inexact TME, using Euler-Poincaré reduction theory, and also using classical calculus of variations, were derived recently, and communicated to us by Ratnanather, Baigent, Mumford and Miller (2000). An early version without the use of Euler-Poincaré theory appears in Mumford (1998b). We should note that although Trouvé (1995) does not mention Euler-Poincaré reduction, and does not give a $\mathrm{PDE}$ for template matching explicitly, he was certainly aware of, and used the idea of moving back and forth between the tangent space at identity and a general point of $\operatorname{Diff}(M)$. For completeness, we now give the Euler-Poincaré derivation of the $\mathrm{L}^{2}$-TME in our notation. We do the derivation of the exact equations, i.e. it is assumed that the two images being compared are diffeomorphic.

\subsection{Derivation of Exact $\mathrm{L}^{2}$-TME}

We have seen in Claim 1 that the $\mathrm{L}^{2}$ metric of Definition 3 is right invariant. Thus if we define a Lagrangian $L: T(\operatorname{Diff}(M)) \rightarrow \mathbb{R}$ as

$$
L(\varphi(t), \dot{\varphi}(t))=\frac{1}{2}(\dot{\varphi}(t), \dot{\varphi}(t))_{\mathrm{L}^{2}}
$$

then it will also be right invariant under that action of $\operatorname{Diff}(M)$.

Let $\varphi:[0,1] \rightarrow \operatorname{Diff}(M)$ be a smooth parameterized curve in $\operatorname{Diff}(M)$ between the points $e=\varphi(0)$ (identity map) and $\varphi(1) \in \operatorname{Diff}(M)$. The point $\varphi(1)$ is such that $f=g \circ(\varphi(1))$ for the given images $f$ and $g$. Take a smooth family of curves $\varphi_{\epsilon}$ with the same end points $\varphi(0)$ and $\varphi(1)$ and such that $\varphi_{0}=\varphi$. Define the variations of the curve $\varphi$ to be the vector field $\delta \varphi=d /\left.d \epsilon\right|_{\epsilon=0} \varphi_{\epsilon}$ along $\varphi$.

Consider the variational principle $\delta \int_{0}^{1} L(\varphi(t), \dot{\varphi}(t)) d t=0$, with the above variations. By the discussion in Section 3.2 the solution of the variational principle above is a geodesic on $\operatorname{Diff}(M)$ under the $\mathrm{L}^{2}$ metric from the identity map $e \in \operatorname{Diff}(M)$ to the diffeomorphism $\varphi(1)$ which satisfies the condition of matching, i.e. $f=g \circ(\varphi(1))$. Due to the right invariance of the Lagrangian we can use the Euler-Poincaré reduction theorem (see Theorem 13.5.3, Page 437 of Marsden and Ratiu (1999) and Section 3.3 of this paper). By applying this theorem, we will get a variational principle on $\mathfrak{X}(M)$ (called the reduced variational principle) and hence a differential equation in terms of Eulerian veclocity vector fields on $M$. These are the exact $\mathrm{L}^{2}$-TME derived by Ratnanather, Baigent, Mumford and Miller (2000).

The reduced variational principle uses the reduced Lagrangian $l$ which is a function on the tangent space at identity of $\operatorname{Diff}(M)$, namely on $\mathfrak{X}(M)$, the space of all spatial or Eulerian velocity vector fields on $M$. As noted in Section 3.3 the function $l$ is just the restriction of $L$ to $T_{e}(\operatorname{Diff}(M))$. Furthermore, $L$ as defined in equation (4) is right invariant under right action of $\operatorname{Diff}(M)$. As a result $L(\varphi, \dot{\varphi})=L\left(e, \dot{\varphi} \circ \varphi^{-1}\right)=l(\boldsymbol{u})$ where $\boldsymbol{u}=\dot{\varphi} \circ \varphi^{-1}$. Thus by Definition 3

$$
l(\boldsymbol{u})=\frac{1}{2} \int_{M}\|\dot{\varphi}(X, t)\|^{2} J\left(\varphi_{t}\right)(X) d X,
$$


where the overdot is time derivative keeping $X$ fixed and the norm $\|\cdot\|$ is the standard norm in $\mathbb{R}^{n}$. Let $X=\varphi_{t}^{-1}(x)$. Then $d X=J\left(\varphi_{t}^{-1}\right)(x) d x$. Thus,

$$
\begin{aligned}
l(\boldsymbol{u}) & =\frac{1}{2} \int_{M}\left\|\dot{\varphi}\left(\varphi_{t}^{-1}(x), t\right)\right\|^{2} J\left(\varphi_{t}\right)\left(\varphi_{t}^{-1}(x)\right) J\left(\varphi_{t}^{-1}\right)(x) d x \\
& =\frac{1}{2} \int_{M}\|\boldsymbol{u}(x, t)\|^{2} d x=\frac{1}{2}\|\boldsymbol{u}\|_{\mathrm{L}^{2}}^{2} .
\end{aligned}
$$

Let us call the functional for the reduced variational principle $\mathcal{E}$, where

$$
\mathcal{E}(\boldsymbol{u})=\int_{0}^{1} l(\boldsymbol{u}(t)) d t=\frac{1}{2} \int_{0}^{1}\|\boldsymbol{u}(x, t)\|_{\mathrm{L}^{2}}^{2} d t .
$$

Then $\delta \mathcal{E}=\int_{0}^{1} \int_{M}\langle\boldsymbol{u}(x, t), \delta \boldsymbol{u}(x, t)\rangle d x d t$, where the inner product inside the integral is the usual dot product in $\mathbb{R}^{n}$. Inserting the definition of $\delta \boldsymbol{u}$ from Section (3.3) in this integral and setting the resulting expression to 0 we get

$$
\int_{0}^{1} \int_{M}\left\langle\boldsymbol{u}(x, t), \dot{\boldsymbol{w}}(x, t)+[\boldsymbol{w}, \boldsymbol{u}]_{L}(x, t)\right\rangle d x d t=0
$$

Now substitute the definition of $[\boldsymbol{w}, \boldsymbol{u}]_{L}$ from Section 3.3, or page 20 of Marsden and Ratiu 1999. With this the above equation becomes

$$
\int_{0}^{1} \int_{M}\langle\boldsymbol{u}, \dot{\boldsymbol{w}}\rangle-\langle\boldsymbol{u},(\boldsymbol{w} \cdot \nabla) \boldsymbol{u}\rangle+\langle\boldsymbol{u},(\boldsymbol{u} \cdot \nabla) \boldsymbol{w}\rangle d x d t=0
$$

Using integration by parts on the time variable for the first term, and the fact that $\boldsymbol{w}(x, 0)=\boldsymbol{w}(x, 1)=0 \forall x \in M$ (see Section 3.3) implies that

$$
\int_{0}^{1} \int_{M}\langle\boldsymbol{u}(x, t), \dot{\boldsymbol{w}}(x, t)\rangle d x d t=-\int_{0}^{1} \int_{M}\langle\dot{\boldsymbol{u}}(x, t), \boldsymbol{w}(x, t)\rangle d x d t
$$

For the second and third term of equation (5) also, the goal is to rewrite those in the form $\langle\cdot, \boldsymbol{w}\rangle$. To bring the second term into the required form, note that $(\boldsymbol{w} \cdot \nabla) \boldsymbol{u}=\mathbf{D} \boldsymbol{u} \cdot \boldsymbol{w}$ where $\mathbf{D}$ denotes the spatial derivative. Thus

$$
\langle\boldsymbol{u},(\boldsymbol{w} \cdot \nabla) \boldsymbol{u}\rangle=\langle\boldsymbol{u}, \mathbf{D} \boldsymbol{u} \cdot \boldsymbol{w}\rangle=\left\langle(\mathbf{D} \boldsymbol{u})^{T} \cdot \boldsymbol{u}, \boldsymbol{w}\right\rangle .
$$

For the third term, we use Fact 6 . From Fact $\left.2 \boldsymbol{u}\right|_{\partial M}=0$ and so the RHS of equation (1) is 0 . Thus

$$
\int_{M}\langle\boldsymbol{u},(\boldsymbol{u} \cdot \nabla) \boldsymbol{w}\rangle d x=-\int_{M} \operatorname{div} \boldsymbol{u}\langle\boldsymbol{w}, \boldsymbol{u}\rangle+\langle(\boldsymbol{u} \cdot \nabla) \boldsymbol{u}, \boldsymbol{w}\rangle d x .
$$

Using equations (6), (7) and (8) in equation (5) one gets that

$$
\int_{0}^{1} \int_{M}-\langle\dot{\boldsymbol{u}}, \boldsymbol{w}\rangle-\left\langle(\mathbf{D} \boldsymbol{u})^{T} \cdot \boldsymbol{u}, \boldsymbol{w}\right\rangle-\langle(\operatorname{div} \boldsymbol{u}) \boldsymbol{u}, \boldsymbol{w}\rangle-\langle(\boldsymbol{u} \cdot \nabla) \boldsymbol{u}, \boldsymbol{w}\rangle d x d t=0
$$


Then since $\boldsymbol{w}$ is arbitrary it follows that

$$
\frac{\partial \boldsymbol{u}}{\partial t}+(\mathbf{D} \boldsymbol{u})^{T} \cdot \boldsymbol{u}+(\operatorname{div} \boldsymbol{u}) \boldsymbol{u}+(\boldsymbol{u} \cdot \nabla) \boldsymbol{u}=0
$$

The above equation (9) is the template matching equation, for exact matching, i.e. the $\mathrm{L}^{2}-\mathrm{TME}$, as communicated to us by Ratnanather, Baigent, Mumford, Miller (2000). We have just repeated the derivation in our notation for completeness. Note that for all $(x, t) \in M \times[0,1],(\mathbf{D} \boldsymbol{u}(x, t))^{T} \cdot \boldsymbol{u}(x, t)=\frac{1}{2} \nabla\left(\|\boldsymbol{u}(x, t)\|^{2}\right)$ where now, the norm on the RHS is the standard norm in $\mathbb{R}^{n}$. With this, the $\mathrm{L}^{2}$-TME, equation (9) can be written in an alternative form as

$$
\frac{\partial \boldsymbol{u}}{\partial t}+(\operatorname{div} \boldsymbol{u}) \boldsymbol{u}+(\boldsymbol{u} \cdot \nabla) \boldsymbol{u}=-\frac{1}{2} \nabla\left(\|\boldsymbol{u}\|^{2}\right)
$$

where $\boldsymbol{u}=\boldsymbol{u}(x, t)$ is the unknown time dependent spatial (Eulerian) velocity vector field on $M$ that vanishes on the boundary $\partial M$ of $M$.

These equations can be written more concisely, in the Lie derivative form as $\partial \beta / \partial t+£_{\boldsymbol{u}} \beta=0$ where $\beta$ is the one form density associated with $\boldsymbol{u}$ and where $£$ is the Lie derivative. In $\mathbb{R}^{n}, \beta=\sum_{i=1}^{n} u^{i} d x^{i} \otimes d^{n} x$. These are the Euler-Poincaré equations associated with the right invariant $\mathrm{L}^{2}$ metric of Definition 3 on the diffeomorphism group. The advantage of this form is that it can accomodate other metrics, simply by changing $\beta$. This will become clear when we derive the $\mathrm{H}_{\alpha}^{1}$-ATME in Section 6.1.

\section{Averaged Template Matching Equations}

We now derive $\mathrm{H}_{\alpha}^{1}$-ATME, which are a set of averaged template matching equations. These equations are analogs of the exact template matching equations that retain all the geometric properties associated with the diffeomorphism group and which are expected to average out small scale features and so should, as in hydrodynamics, be more computationally efficient for resolving the larger scale features. From a geometric point of view, the new equations may be viewed as coming from a change in norm that is used to measure the distance between images.

\subsection{Derivation of $\mathrm{H}_{\alpha}^{1}$-ATME}

The steps in deriving the $\mathrm{H}_{\alpha}^{1}$-ATME are almost identical to those used for $\mathrm{L}^{2}$ TME, except that we use the $\mathrm{H}_{\alpha}^{1}$ metric (Definition 4) on Diff $(M)$, instead of the $\mathrm{L}^{2}$ metric. Thus we start with a Lagrangian $L: T(\operatorname{Diff}(M)) \rightarrow \mathbb{R}$ defined as

$$
L(\varphi(t), \dot{\varphi}(t))=\frac{1}{2}(\dot{\varphi}(t), \dot{\varphi}(t))_{\mathrm{H}_{\alpha}^{1}}
$$

The initial and final value conditions are the same as in the $\mathrm{L}^{2}-\mathrm{TME}$ case, i.e. $f=g \circ(\varphi(1))$. By Claim 2 this Lagrangian is invariant under the right action of 
$\operatorname{Diff}(M)$. Thus, as in Section 5.1, Euler-Poincaré reduction can be applied but with a different norm. The reduced Lagrangian is $l(\boldsymbol{u})=\frac{1}{2}\|\boldsymbol{u}\|_{\mathrm{H}^{1}}^{2}$. By Definition 4 of the $\mathrm{H}_{\alpha}^{1}$ norm, this implies that $l(\boldsymbol{u})=\frac{1}{2} \int_{M}\langle\boldsymbol{u}, \boldsymbol{u}\rangle+\alpha^{2} \sum_{i=1}^{n}\left\langle\mathbf{D}_{i} \boldsymbol{u}, \mathbf{D}_{i} \boldsymbol{u}\right\rangle d x$. The functional that appears in the reduced variational principle is

$$
\mathcal{E}(\boldsymbol{u})=\int_{0}^{1} l(\boldsymbol{u}) d t=\frac{1}{2} \int_{0}^{1} \int_{M}\langle\boldsymbol{u}, \boldsymbol{u}\rangle+\alpha^{2} \sum_{i=1}^{n}\left\langle\mathbf{D}_{i} \boldsymbol{u}, \mathbf{D}_{i} \boldsymbol{u}\right\rangle d x d t
$$

Let $\boldsymbol{u}_{\epsilon}$ be a one parameter family of spatial vector fields on $M$, depending smoothly on $\epsilon$ such that $\boldsymbol{u}_{0}=\boldsymbol{u}$ and as usual $\delta \boldsymbol{u} \equiv \partial \boldsymbol{u}_{\epsilon} /\left.\partial \epsilon\right|_{\epsilon=0}$. The variations $\delta \mathcal{E}(\boldsymbol{u})$ are given by

$$
\delta \mathcal{E}(\boldsymbol{u})=\int_{0}^{1}\left\langle\frac{\delta l}{\delta \boldsymbol{u}}, \delta \boldsymbol{u}\right\rangle d t
$$

We now compute the above expression. The integrand is

$$
\left.\left\langle\frac{\delta l}{\delta \boldsymbol{u}}, \delta \boldsymbol{u}\right\rangle \equiv \frac{d}{d \epsilon}\right|_{\epsilon=0} l\left(\boldsymbol{u}_{\epsilon}\right)=\int_{M}\left\langle\boldsymbol{u}_{\epsilon}, \frac{\partial \boldsymbol{u}_{\epsilon}}{\partial \epsilon}\right\rangle+\left.\alpha^{2} \sum_{i=1}^{n}\left\langle\frac{\partial \boldsymbol{u}_{\epsilon}}{\partial x_{i}}, \frac{\partial^{2} \boldsymbol{u}_{\epsilon}}{\partial \epsilon \partial x_{i}}\right\rangle\right|_{\epsilon=0} d x
$$

But

$$
\begin{aligned}
\sum_{i=1}^{n}\left\langle\frac{\partial \boldsymbol{u}_{\epsilon}}{\partial x_{i}}, \frac{\partial^{2} \boldsymbol{u}_{\epsilon}}{\partial \epsilon \partial x_{i}}\right\rangle & =\sum_{i=1}^{n} \sum_{j=1}^{n} \frac{\partial u_{\epsilon}^{j}}{\partial x_{i}} \frac{\partial^{2} u_{\epsilon}^{j}}{\partial \epsilon \partial x_{i}} \\
& =\sum_{j=1}^{n} \sum_{i=1}^{n} \frac{\partial u_{\epsilon}^{j}}{\partial x_{i}} \frac{\partial^{2} u_{\epsilon}^{j}}{\partial \epsilon \partial x_{i}}=\sum_{j=1}^{n}\left\langle\frac{\partial u_{\epsilon}^{j}}{\partial x} \frac{\partial^{2} u_{\epsilon}^{j}}{\partial x \partial \epsilon}\right\rangle
\end{aligned}
$$

Now

$$
\left.\int_{M} \sum_{i=1}^{n}\left\langle\frac{\partial u_{\epsilon}^{i}}{\partial x}, \frac{\partial^{2} u_{\epsilon}^{i}}{\partial x \partial \epsilon}\right\rangle\right|_{\epsilon=0} d x=\int_{M} \sum_{i=1}^{n}\left\langle\frac{\partial u^{i}}{\partial x}, \frac{\partial\left(\delta u^{i}\right)}{\partial x}\right\rangle d x .
$$

Then using integration by parts,

$$
\begin{aligned}
\int_{M}\left\langle\frac{\partial u^{i}}{\partial x}, \frac{\partial\left(\delta u^{i}\right)}{\partial x}\right\rangle d x & \equiv \int_{M} \sum_{j=1}^{n} \frac{\partial u^{i}}{\partial x_{j}} \frac{\partial\left(\delta u^{i}\right)}{\partial x_{j}} d x \\
& =-\int_{M} \sum_{j=1}^{n} \frac{\partial^{2} u^{i}}{\partial x_{j}^{2}} \delta u^{i} d x+\int_{\partial M} \sum_{j=1}^{n} \frac{\partial u^{i}}{\partial x_{j}} \delta u^{i} \nu^{j} d S
\end{aligned}
$$

where $\nu=\left(\nu^{1}, \ldots, \nu^{n}\right)$ is the outward pointing normal field on the boundary $\partial M$ of $M$. But by Fact $2,\left.\boldsymbol{u}\right|_{\partial M}=0$ so we get that

$$
\int_{M}\left\langle\frac{\partial u^{i}}{\partial x}, \frac{\partial\left(\delta u^{i}\right)}{\partial x}\right\rangle d x=-\int_{M} \sum_{j=1}^{n} \frac{\partial^{2} u^{i}}{\partial x_{j}^{2}} \delta u^{i} d x=-\int_{M}\left(\Delta u^{i}\right) \delta u^{i} d x .
$$


Thus

$$
\left\langle\frac{\delta l}{\delta \boldsymbol{u}}, \delta \boldsymbol{u}\right\rangle=\int_{M}\langle\boldsymbol{u}, \delta \boldsymbol{u}\rangle-\alpha^{2} \sum_{i=1}^{n}\left(\Delta u^{i}\right) \delta u^{i} d x
$$

Substituting this into equation (12) we get that

$$
\begin{aligned}
\delta \mathcal{E}(\boldsymbol{u}) & =\int_{0}^{1} \int_{M}\langle\boldsymbol{u}, \delta \boldsymbol{u}\rangle-\alpha^{2} \sum_{i=1}^{n}\left(\Delta u^{i}\right) \delta u^{i} d x d t \\
& =\int_{0}^{1} \int_{M}\left\langle\boldsymbol{u}-\alpha^{2} \Delta \boldsymbol{u}, \delta \boldsymbol{u}\right\rangle d x d t
\end{aligned}
$$

Now substituting the expression for $\delta \boldsymbol{u}$ from Section 3.3 we get

$$
\delta \mathcal{E}(\boldsymbol{u})=\int_{0}^{1} \int_{M}\left\langle\boldsymbol{u}-\alpha^{2} \Delta \boldsymbol{u}, \dot{\boldsymbol{w}}-(\boldsymbol{w} \cdot \nabla) \boldsymbol{u}+(\boldsymbol{u} \cdot \nabla) \boldsymbol{w}\right\rangle d x d t
$$

We move the derivative operators away from $\boldsymbol{w}$, as in the $\mathrm{L}^{2}$-TME derivation in Section 5.1 , to get an integrand of the form $\langle\cdot, \boldsymbol{w}\rangle$. Because of the arbitrariness of $\boldsymbol{w}$, we get the isotropic averaged $\mathrm{H}_{\alpha}^{1}$ template matching equations, i.e. the $\mathrm{H}_{\alpha}^{1}-\mathrm{ATME}$ as

$$
\begin{aligned}
\frac{\partial}{\partial t} \boldsymbol{u}-\alpha^{2} \frac{\partial}{\partial t} \Delta \boldsymbol{u}+\boldsymbol{u}(\operatorname{div} \boldsymbol{u})-\alpha^{2}(\operatorname{div} \boldsymbol{u}) \Delta \boldsymbol{u}+(\boldsymbol{u} \cdot \nabla) \boldsymbol{u}- \\
\alpha^{2}(\boldsymbol{u} \cdot \nabla) \Delta \boldsymbol{u}+(\mathbf{D} \boldsymbol{u})^{T} \cdot \boldsymbol{u}-\alpha^{2}(\mathbf{D} \boldsymbol{u})^{T} \cdot \Delta \boldsymbol{u}=0
\end{aligned}
$$

where $\Delta$ is the componentwise Laplacian and $\mathbf{D} \boldsymbol{u}$ is the spatial derivative of $\boldsymbol{u}$. Here $\boldsymbol{u}=\boldsymbol{u}(x, t)$ is the unknown time dependent spatial (Eulerian) velocity vector field on $M$ which vanishes on the boundary $\partial M$ of $M$. To make it easier to see the relationship between the structure of the $\mathrm{H}_{\alpha}^{1}$-ATME and $\mathrm{L}^{2}$-TME we define $\boldsymbol{v} \equiv\left(1-\alpha^{2} \Delta\right) \boldsymbol{u}$. Then the $\mathrm{H}_{\alpha}^{1}$-ATME equation (13) becomes

$$
\frac{\partial \boldsymbol{v}}{\partial t}+(\mathbf{D} \boldsymbol{u})^{T} \cdot \boldsymbol{v}+(\operatorname{div} \boldsymbol{u}) \boldsymbol{v}+(\boldsymbol{u} \cdot \nabla) \boldsymbol{v}=0
$$

The Lie derivative form of these equations is $\partial \beta / \partial t+£_{\boldsymbol{u}} \beta=0$ where now $\beta$ is the one form density associated with $\boldsymbol{v}=\left(1-\alpha^{2} \Delta\right) \boldsymbol{u}$.

\section{Connections With Fluid Mechanics}

We now give the analogy and connections with fluid mechanics which have inspired our present work. We start with the connection between TME and fluid mechanics. The $\mathrm{L}^{2}$-TME in $n$ spatial dimensions, namely equation (9) or equivalently (10), is a higher dimensional analogue of the inviscid Burger's equation. By this we mean that in one spatial dimension the $\mathrm{L}^{2}$-TME reduce to the equation $u_{t}+3 u u_{x}=0$ where the subscripts indicate derivatives and $u=u(x, t)$ is the 
velocity of the fluid at location $x$ at time $t$. This fact is mentioned in Mumford (1998). Burger's equation, at least in the initial value formulation, can develop shocks. The framework for $\mathrm{L}^{2}$-TME sets this equation in higher dimensions, and as an initial-final value problem. It is possible that shocks may develop in this formulation also. The well-posedness and possibility of shocks in $\mathrm{L}^{2}$-TME remain to be checked. However, as in the case of Burger's equation and incompressible fluid mechanics, they are well-posed for short time evolution; see Marsden, Ratiu and Shkoller (2000) and Marsden and Shkoller (2001) and references therein.

Our $\mathrm{H}_{\alpha}^{1}$-ATME, on the other hand, contain diffusive terms which may ameliorate some of the analytical problems of $\mathrm{L}^{2}-\mathrm{TME}$, and may be a reason to expect more stable numerics. In one spatial dimension our $\mathrm{H}_{\alpha}^{1}$-ATME, equations (13) reduce to the shallow water equations $u_{t}-u_{x x t}=-3 u u_{x}+2 u_{x} u_{x x}+u u_{x x x}$ or equivalently $v_{t}+u v_{x}+2 v u_{x}=0$ where $v=u-u_{x x}$ (here $\alpha$ of $\mathrm{H}_{\alpha}^{1}$-ATME has been set to unity). These equations are completely integrable and have peaked solitons, as was shown by Camassa and Holm (1993). The shallow water equations, like Burger's equation, also have a smooth spray, as was shown by Shkoller (1998) and hence one has local existence and uniqueness of geodesics. The $\mathrm{L}^{2}$ TME are equations of geodesics on $\operatorname{Diff}(M)$ under the right invariant $\mathrm{L}^{2}$ metric whereas the $\mathrm{H}_{\alpha}^{1}$-ATME are equations of geodesics under a right invariant $\mathrm{H}_{\alpha}^{1}$ metric.

Recently, averaged Euler and Navier-Stokes equations for fluid mechanics have been developed. See for example Holm, Marsden and Ratiu (1998) and Marsden, Ratiu and Shkoller (2000). Besides having nice analytical properties, preliminary numerical experiments (see Mohseni et al (2000) and references therein) of these averaged Euler and Navier-Stokes equations show the possible advantages of an averaging approach in the context of numerical solution of nonlinear equations of fluid mechanics.

There is however a very important difference between the template matching framework and the usual fluid mechanics framework. In template matching the equations have to be solved as an initial-final value problem. Thus one is given image $f$ and it has to be deformed to image $g$ moving the pixels in such a way that the motion of the image during the deformation satisfies the template matching equations. In fluid mechanics one is typically interested in giving some initial velocity and studying how the particles move.

\section{Discussion and Future Work}

We have named the $\mathrm{H}_{\alpha}^{1}$ equations averaged equations. This is with the expectation that they will have averaging properties like the averaged equations of hydrodynamics described in Marsden and Shkoller (2001). But we have not yet done an averaging derivation in the template matching context to see if the solution does indeed allow one to compare images while ignoring features smaller than $\alpha$. This is the most important task that remains to be done. Furthermore, a condition like $f=g \circ(\varphi(1))$ will have to be modified in the averaging case possibly by preprocessing $f$ and $g$. 
It is our expectation that there will be considerable improvement in the numerical performance of the $\mathrm{H}_{\alpha}^{1}$-ATME. This expectation is based on the analogous situation in fluid dynamics in which averaging improves numerical simulation as demonstrated in Mohseni et. al (2000) and references therein. We also plan to investigate generalizations of the procedures here using inexact matching and semidirect product theory (used for inhomogeneous fluid problems, for example), to generalize beyond binary images. We also want to explore the role of the rotation group and reduction constructions. This is based on the fact that the notion of shape space is a technique common to both mechanics and computer vision.

Due to an initial-final value formulation, it appears that an optimization approach is one way to solve template matching type equations, as is done in Trouvé $(1995,1998)$. Thus when it comes to computations, the PDEs of template matching (9) or (13) may, or may not be used directly for some applications. In the optimization approach, one goes back to the Lagrangian (material) formulation on the group and finds geodesics directly, by a gradient descent type algorithm for example.

One benefit of the PDE formulation is theoretical, now one knows what equation is being solved. But more importantly, we emphasize that the very reason that we were able to arrive at an averaged version of $\mathrm{L}^{2}$-TME was because we knew how averaging worked in hydrodynamics by changing the metric on the group $\operatorname{Diff}(M)$, and we knew how to go back and forth between an arbitrary point on the group and identity using Euler-Poincaré theory. Finally, there may be other problems within computer vision or outside it, in which the PDEs are used directly in the computations.

As for the existence of minimizer of the energy functional, Trouvé (1995, 1998) uses a different definition of distance on $\operatorname{Diff}(M)$. He gives a sufficient condition on the metric on $\operatorname{Diff}(M)$ for the variational problem of template matching to have a minimizer. Roughly speaking, this condition (which is part of what he calls the admissibility criteria) is that the metric must be as strong as the $C^{1}$ metric. By Sobolev embedding theorem this implies that an $H^{s}$ metric will satisfy this condition iff $s>n / 2+1$. Thus for $n=2$ or 3 , an $H^{1}$ or $H^{2}$ metric, including the $\mathrm{H}_{\alpha}^{1}$ metric will not satisfy this condition. However, note that Trouvé's admissibility criteria is a sufficient condition. In practice in template matching sometimes the metric $\int_{M}\langle\boldsymbol{u}, \boldsymbol{u}\rangle+(\Delta \boldsymbol{u})^{2} d x$ is used as mentioned in Grenander and Miller (1998). This metric also does not satisfy the admissibility criteria. This suggests that more work on the theory of existence of minimizer for template matching functionals is needed.

Finally we note that we have only shown that $\mathrm{d}_{\mathcal{P}}$ is a pseudometric. If the infimum in Definition 2 in not achieved $\mathrm{d}_{\mathcal{P}}$ is only a pseudometric and there can exist $f$ and $g, f \neq g$, but $\mathrm{d}_{\mathcal{P}}(f, g)=0$. What do specific examples (if any) of such $f, g$ pairs look like ? Even more interestingly one can ask if for some metrics on Diff $(M)$, for example for the $\mathrm{H}_{\alpha}^{1}$ metric of Definition $4, \mathrm{~d}_{\mathcal{P}}$ is a metric. We plan to investigate these questions. 
Acknowledgements We would like to thank J. Tilak Ratnanather for bringing the TME to our attention in 1999 and keeping us informed of their work since then. We would also like to thank Steve Shkoller, Antonio Hernandez, Sergey Pekarsky and Banavara Shashikanth for helpful discussions. Comments from the mutual review group in Caltech Computer Science, especially the detailed suggestions from Min Chen were very useful in improving the organization of the paper.

\section{References}

Abraham, R., Marsden, J. E. and Ratiu, T. S.: Manifolds, Tensor Analysis and Applications. Springer Verlag (1988)

Camassa, R. and Holm D. D.: An integrable shallow water equation with peaked solitons. Phys. Rev. Lett. 71 (1993) 1661-1664

Dupuis, P., Grenander, U. and Miller, M. I.: Variational problems on flows of diffeomorphisms for image matching. Quarterly of Applied Mathematics LVI (1998) $587-600$

Ebin, D. E., Marsden, J. E.: Groups of diffeomorphisms and the motion of an incompressible fluid. Ann. of Math. 92 (1970) 102-163

Grenander, U. and Miller, M. I.: Computational anatomy: an emerging discipline. Quarterly of Applied Mathematics LVI (1998) 617-694

Hirani, A. N., Marsden, J. E. and Arvo, J.: Averaged template matching equations. California Institute of Tecnology, Computer Science Technical Report (2001)

Holm, D. D., Marsden, J. E. and Ratiu, T. S.: Euler-Poincaré models of ideal fluids with nonlinear dispersion. Phys. Res. Lett. 349 (1998) 4173-4177

Marsden, J. E. and Ratiu, T. S.: Introduction to Mechanics and Symmetry, Second Edition. Springer Verlag (1999)

Marsden, J. E., Ratiu, T. S. and Shkoller, S.: The geometry and analysis of the averaged Euler equations and a new diffeomorphism group. Geometric and Functional Analysis 10 (2000) 582-599

Marsden, J. E. and Shkoller, S.: Global well-posedness of the isotropic LANS equations. Proc. Royal Soc. London (to appear)

Miller, M. I. and Younes, L.: Group actions, homeomorphisms and matching : a general framework. Preprint (1999)

Mohseni, K., Shkoller, S., Kosović, B., Marsden, J. E., Carati, D., Wray, A. and Rogallo, R.: Numerical Simulations of Homogeneous Turbulence using the LagrangianAveraged Navier-Stokes Equations. Proc. CTR summer school (2000)

Mumford, D.: Unpublished note (1998)

Mumford, D.: Pattern theory and vision. Questions Mathématiques en Traitement du Signal et de L'Image, Institut Henri Poincaré (1998) 7-13

Ratnanather, J. T., Baigent, S. A., Mumford, D. and Miller, M. I.: Personal communication (2000)

Shkoller, S.: Geometry and curvature of diffeomorphism groups with $H^{1}$ metric and mean hydrodynamics. J. Funct. Anal. 160 (1998) 337-365

Trouvé, A.: An infinite dimensional group approach for physics based models in pattern recognition. Preprint (1995)

Trouvé, A.: Diffeomorphism groups and pattern matching in image analysis. Intl. J. Comp. Vis. 28 (1998) 213-221

Younes, L.: Deformations, warping and object comparison. Preprint (2000) 